\title{
Research on the Aerodynamics Loads of Wind Turbine Blades
}

\author{
Zhiqiang $\mathrm{Xu}$ \\ Department of Physics and Technology, Kunming University, Kunming 650214, China \\ 2793436659@qq.com
}

Keywords: The Strip Theory; The Equilibrium Wake; The Dynamic Inflow; Aerodynamic Load

\begin{abstract}
A more precise strip theory is adopt for the fist time in calculating the aerodynamic loads ,considering the elevation of the wind wheel spindle, the shearing of wind and the tilt angle of the direction when blades brandish. Subsequently, the dynamic inflow model will be considered separately on the basis of balancing the wake, calculating the unsteady aerodynamic inducible factor. In the sectional coordinate system of the blade of the wind turbine, the aerodynamic loads of the equilibrium wake, inflow dynamic and dynamic stall are calculated separately. The loads of the calculation under the three models are compared. In addition, the influence of three kinds of aerodynamic model on the calculation of the aerodynamic loads is analyzed.
\end{abstract}

\section{Introduction}

Energy and environment have become major issues for sustainable development, which has increasingly attracted wide attention from the international community. As an important renewable energy, wind energy is clean, non-polluting, safe and abundant. Therefore, it has received wide attention around the world. It not only develops fastest among the growing renewable energy, but also has become the renewable energy which will develop in a large scale. In recent years, the wind power industry is developing rapidly throughout the world. The wind power generation capacity in China has surpassed the nuclear power, making it the third largest source of electricity. Both the wind power technology and the wind power industry have made considerable progress. However, the design and manufacturing technology of large wind turbines are still in its infancy, with independent innovation and weak capability, lacking of practice experience. Besides, with the increasing demand for energy and the increase of enforcement of environmental regulations, through technical innovation, design, manufacturing process improvement and cost reduction, the advantages economy of the wind power technology will become increasingly apparent as the continuous development and improvement of multi-disciplinary high-tech.

\section{The Blade Element Momentum Theory}

The aerodynamics of the wind turbine blades mainly solves the calculating of aerodynamic performance of the wind turbine blades and shows the relationship between the calculating of the aerodynamic performance of the wind turbine blades and its aerodynamic shape. Nowadays, the $p$ the strip theory is adopted to calculate the aerodynamic performance of the wind turbine and men while a variety of factors which affect the aerodynamic performance of the wind turbine should be considered and the model should be corrected.. In the actual runtime, the wind turbine is also affected by many factors, such as wind shear, yaw, the structural parameters of the wind turbine and the installation parameters of the wind turbine. The calculation of the aerodynamic performance is an important part in a wind turbine design and check. After the aerodynamic shape of the blade is designed and its aerodynamic performance is calculated, which can be evaluated as a result of the design; conversely, the results of the aerodynamic performance can be used as feedback, providing data for the correction of the aerodynamic shape of the paddle. Of several aerodynamics analysis methods of the wind turbine, the blade element momentum theory may achieve a good compromise on the accuracy and computational efficiency model. It has been more widely used in design and aerodynamic calculating of the wind turbine blade. The theory combines the theory of momentum and the blade element theory. The aerodynamic performance of the wind turbine blades can be 
calculated more accurately [2] [3]. To calculate the aerodynamic forces and moments on the blade of the wind turbine, the axial velocity inducible factor a of wind wheel rotating surface and a tangential velocity inducing factor $\alpha^{\prime}$ must be firstly obtained. It can be integrated by using momentum theory and blade element theory. The formulae are shown as formula 1 and 2 [1]

$$
\begin{gathered}
\alpha=\frac{B c\left(C_{L} \cos \phi+C_{D} \sin \phi\right)}{8 \pi r \sin ^{2} \phi+B c\left(C_{L} \cos \phi+C_{D} \sin \phi\right)} \\
\alpha^{\prime}=\frac{B c\left(C_{L} \sin \phi-C_{D} \cos \phi\right)}{8 \pi r \sin \phi \cos \phi-B c\left(C_{L} \sin \phi-C_{D} \cos \phi\right)}
\end{gathered}
$$

$B$ is the number of blades, $c$ is a partial chord length, $t$ is the volume control on the radial position, $C_{L}, C_{D}$ are respectively for lift and drag coefficients of airfoil blade element, the inflow angle is expressed as

$$
\phi=\tan ^{-1} \frac{(1-\alpha) U}{\left(1+\alpha^{\prime}\right) \Omega r}
$$

$\Omega$ is the rotate speed of the wind turbine, making,

$$
\begin{aligned}
& C_{N}=C_{L} \cos \varphi+C_{D} \sin \varphi \\
& C_{T}=C_{L} \sin \varphi-C_{D} \cos \varphi
\end{aligned}
$$

Solidity $\sigma$ is the ratio between the area covered with blades the annular area in a controlled volume:

$$
\sigma=\frac{c B}{2 \pi r}
$$

Formula (2.1), (2.2) can be simplified to:

$$
\begin{gathered}
\alpha=\frac{1}{\frac{4 \sin ^{2} \varphi}{\sigma C_{n}}+1} \\
\alpha^{\prime}=\frac{1}{\frac{4 \sin \varphi \cos \varphi}{\sigma C_{n}}-1}
\end{gathered}
$$

\section{Dynamic Inflow Model Analysis}

There are three kinds of wake models of the wind turbine : balanced wake, frozen wake model and dynamic inflow model [4]. The blade element - momentum theory is based on the assumption of the equilibrium wake. Namely, wake and induced velocity field respond with the change of blades load, which is a quasi-steady-state model [5] In fact, if the wind turbine runs in a very volatile environment, the blade element momentum theory does not apply to the analysis of the pitch angle in the process of the running of the unit, as well as the random variation of the rotate speed and the wind speed. In those cases, the wake of the wind wheel and its induced velocity field will reach a steady state in a delayed period of time. This phenomenon is known as "dynamic inflow. Studies have shown that dynamic inflow effect can sometimes cause the analysis results of the blades waving moment, wind wheel spindle torque and using quasi-steady state model differ by more than $50 \%$ while the blades change the pitch fast. Therefore, considering the effect of dynamic inflow is very important when the load is analyzed.

Figure 1, 2, 3, 4 show how the induced factors change with the wind speed and pitch angle under dynamic inflow model. The horizontal axis represents time. The vertical axis represents the inducible factor- dynamic inflow: $r=54.46 \mathrm{~m}$, -the equilibrium wake: $r=54.46 \mathrm{~m}$. The wind speed variation is that there is a rapid change from $8 \mathrm{~m} / \mathrm{s}$ to $11 \mathrm{~m} / \mathrm{s}$ when the speed reaches $11 \mathrm{~m} / \mathrm{s}$, and 
then change back to $8 \mathrm{~m} / \mathrm{s}$ when it reaches 9 11/s; While the pitch angle variation rapidly changes from 0 degrees to 6 degree $9 \sim 11$ s at the speed of 19 21s and then back to 0 degree at the speed of $19 \sim 21 / s$.

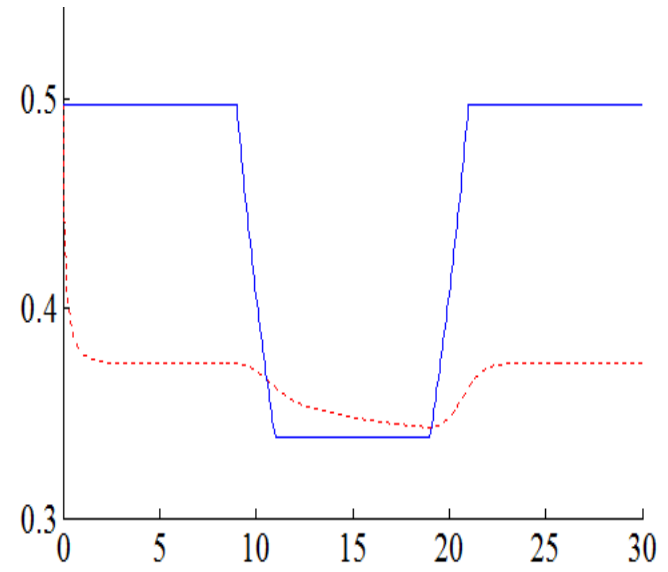

Fig.1 The axial induction factor changes with the wind speed

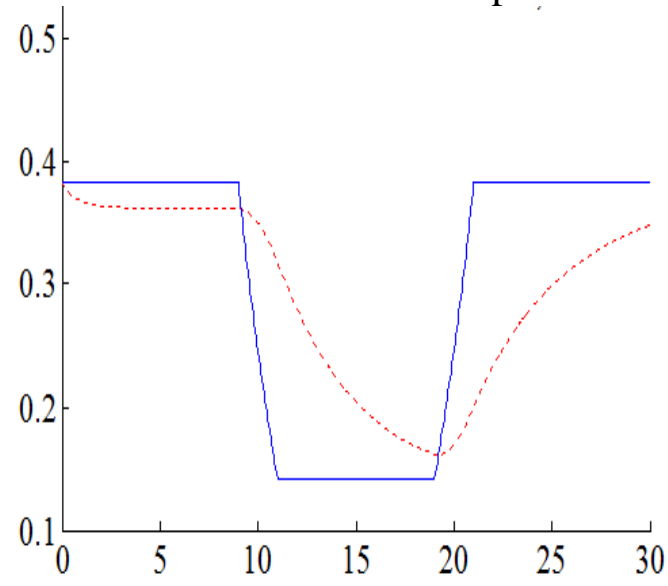

Fig.3 The axial induction factor changes with the pitch angle

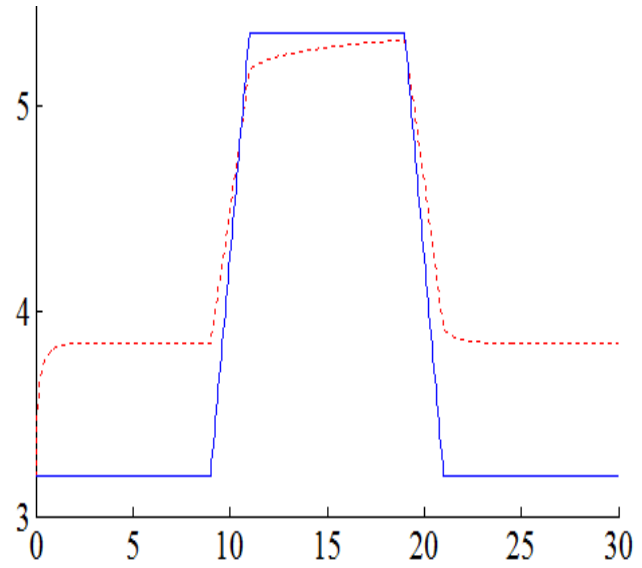

Fig. 2 The tangential induction changes with wind speed

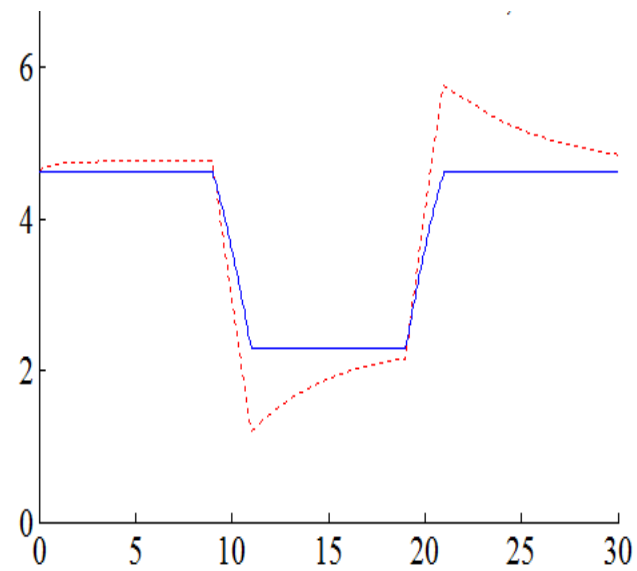

Fig4 The tangential induction factor changes with the pitch angle

Figure 1 and Figure 2 show how the axial inducible factor and the tangential inducible factor change with the wind speed at the cross section of the blade $r=54.46 \mathrm{~m}$ when the speed of wind changes fast. As can be seen from the figure, the inducible factor calculated by the equilibrium wake changes instantaneously while the inducible factor by using the dynamic inflow has a delay and gradual process. Figure 3 and Figure 4 show how the axial and tangential inducible factors change with the pitch angle at the cross section of the blade $r=54.46 \mathrm{~m}$ when the pitch angle changes rapidly. As can be seen from the figure, the dynamic inflow model not only has a gradual process with respect to the equilibrium wake but also correct the larger axial induction factor when the pitch angle changes rapidly.

\section{Conclusion}

Under the equilibrium wake model and the dynamic inflow model, from the calculated axial inducible factor, tangential inducible factor contrast can be seen, the result of the axial inducible factor calculated by the equilibrium wake changes suddenly. But the resulting curve calculated by dynamic inflow model is smooth. This is because the equilibrium wake model is a quasi-steady-state one. It is assumed that the distribution of the induced velocity of the wind wheel is uniform and the induced velocity field of the blade changes with the load, having an effect on the wake immediately; the dynamic inflow is assumed that the wake of the its induced velocity field will reach a steady state in a period of time and there is a delay. 


\section{References}

[1]. Shi liang HAWTThe Analysis of the Unsteady Aerodynamic Characteristics and Structural Dynamic for the Horizontal Axis Wind Turbine[D]. Shantou: Shantou University.

[2]. Lanzafame R, Messina. Fluid dynamics wind turbine design: critical analysis, optimization and application of BEM theory [J]. Renewable Energy, 2007, 32: 2291-2305.

[3]. Moriarty P, Hansen A. AeroDyn Theory Manual [NERL/EL-500-36881][R]. USA: National Renewable Energy Laboratory, 2005.11.

[4]. Wilson R E, Lissaman P B S, Walker S N. Aerodynamic performance of wind turbine[J]. Oregon State University, 1976.

[5]. Xiong Liu, Xianming Zhang, Yan Cheng 2009, 30(4): 412-419The Dynamic Aerodynamic Load Calculation Model of HAWT Based on Dynamic Inflow Theory [J] Solar Academic Journal, 2009, 30(4): 412-419.

[6]. Snel H, Schepers J G. Engineering models for dynamic inflow phenomena [C]. Proceeding of EWEC 91, Netherlands: Elsevier, 1991, 390-396 